\title{
Efficient data analysis approaches to enhance the quality of customer service in Saudi Government sector
}

\author{
Abdullah Baz" \\ Computer Engineering Department, College of Computer and Information Systems, Umm Al-Qura University, \\ Mecca, Saudi Arabia
}

Received: 21-November-2015; Revised: 20-December-2015; Accepted: 08-January-2016

(O2016 ACCENTS

\begin{abstract}
The common aim of all our daily activities is providing services to others or ourselves. Services provided by the government are called public services while those provided by some people to some others are called private services. Both types differ from country to country and from region to region. In Saudi Arabia, public services include education, health, police, trading, and environmental services. It is the aim of all government agencies in Saudi Arabia and other countries around the world to offer more services to their customers, improve the quality of their customer service, and enhance the existing services. Nowadays, most government agencies have already developed a number Information Systems (ISs) to help their customers in applying for services and help their employees in processing the requested services. However, hardly a government agency utilizes the data in the employed ISs to improve the quality of its customer service. This paper is dedicated to addressing this gap by proposing a number of data analysis techniques that can be utilized to improve the quality of customer service and enhance the existing services in the Saudi government sector. Moreover, this article introduced a relational database structure that can be utilized to apply the proposed data analysis techniques.
\end{abstract}

\section{Keywords}

Customer service, Data analysis, Quality of service, Database.

\section{Introduction}

Customer service is the process of providing people with the legitimate need they require, where "Legitimate need" means people's right based upon the rules and regulations of the government and the service provider [1-3]. According to the situations, the same person can require different types of services. If, for example, the customer is a seller of an equipment, then the service might be maintenance to that equipment. If the customer is a patient, then the service is the treatment to his/her illnesses. If the customer is a student, then the service is the education and so on. Based upon the provider, services can be divided into public and private services. The former is provided by the government to all residents or citizens in the country while the latter is provided by some people to some other people, according to a business deal they made [3-7]. Public services differ from country to country and even in some countries, they differ from region to region.

*Author for correspondence
Famous examples of public services include police, health, education, social security, environmental, and trading services.

It is the aim of all service providers to offer more services to their customers, enhance the existing services, and improve the quality of their customer service. This paper discusses this subject in the context of the Saudi government sector, where the tool utilized towards this aim is the Information Technology (IT). The main contributions of this paper are:

1.Proposing a relational database structure the can be utilized by any government agency in Saudi Arabia for the purpose of providing services to their customers.

2.Proposing a number of data analysis techniques that efficiently utilize the services data to enhance the existing services and improve the quality of customer service in government agencies.

The remainder of this paper is organized into six sections. Next section gives the reader the necessary background to this research. Third section introduces the idea and usefulness of Customer Service Information Systems (CSISs). Fourth section 
proposes a relational database structure that can be employed by any Saudi government agency to process their services. Fifth section introduces a number of data analysis techniques that can be utilized to improve the quality of customer service. Section number six summaries the paper and suggests ideas for future work.

\section{Background}

In order to improve customer service, we need first to define the customer service and understand its main components. According to the literature, customer service has three main pillars, which can be described as [3-8]:

1.Service: which is a procedure that comprises one or more steps where each step has a number of inputs, outcomes, and checklist. The Input might include documents and/or information. Outcomes might include progressing the service to the next step, returning it to the previous one, refusing it, and terminating it. The checklist includes checking the customer documents and information in order to take the right decision about the service/step. The service starts if the customer requested it and provided all required inputs of the first step. Each step is assigned to an individual employee or a group of employees to process it. The processing involves inspecting the checklist of each step in order to forward it to the right outcome.

2.Customer: who is the beneficiary eligible to apply for the service and is required to provide the necessary inputs to the service as it progresses.

3.Provider: is the government agency that receives the request and processes it by assigning it to one or more employees.

Based upon the intervention required by the human during processing the service/step, it can be divided into two main categories:

1.Human Intervention Service/Step (HIS): which is a service/step that requires a decision from the authorized employee who process it. This type of service/step requires careful inspection of the available inputs/circumstances in order to choose the right decision about the service/step. Such services/steps cannot be fully automated.

2.None-Human Intervention Service/Step (NHIS): is a service/step that does not require a decision from human. The available information is enough to process the service/step via an automated Information System.

\section{Customer Service Information Systems (CSISs)}

It is the fact that the number of beneficiaries increases day after day in Saudi Arabia, which follows the population growth. Additionally, the number of services they require having the same trend. Therefore, it becomes difficult for the government agencies or any other service providers to process the services manually [3-6]. The best solution to such a problem is to develop an Information System (IS) for the customer to allow them to apply for services and allow the agency to process these services. The said ISs have many names in the academic and industrial societies, including CIS, CRMS, and CSIS (which is used in this research paper). The back end of these ISs is a database that stores the customers, services, \& employees data while the front end is a number of forms that allow the service provider to manipulate these data [5-8]. The manipulating occurs according to the rules and regulations of the government agency. CSISs help the customers in requesting new services, tracking the status of active services, and replying to the queries received from the one who processes the service.

Moreover, CSISs help government agencies in storing of data in a compact form, processing of services in less time \& effort, and validating the received information in an efficient way [5-10]. One of the best examples of such CSISs in Saudi Arabia is SAFEER, which is a web-based IS developed by the Ministry Of Education (MOE). It is dedicated to serving Saudi scholarship holders in any country around the world by providing them with all services they need during the scholarship period.

However, it is worth mentioning that most existing CSISs does not efficiently utilize the services data to improve the quality of customer service or enhance the existing services. The next two sections have dedicated for this purpose. The following section provides an efficient database structure that can be utilized by any government agency in Saudi Arabia in the back end of their CSISs. Furthermore, the section follows the next one proposes a number of data analysis techniques that can be utilized to provide decision makers with the information they need in order to improve the quality of their customer service as well as enhance the existing services. 


\section{Proposed database structure}

Based on the previous two sections, a database structure that serves the business of any government agency can be proposed. The database contains nine tables, which are listed below. Each subsection describes the structure of a table, including all attributes and relations. It is worth mentioning that some tables in the following subsections have relations to some other tables the scope of them are outside the subject of this paper (e.g. Employees, Customers etc.). Hence, the names of these tables are just mentioned and it is assumed they exist in the database. In the text below, PK stands for Primary Key and FK stands for Foreign Key. The proposed tables in the database are:

\subsection{Services}

This table contains the details of all services provided by each government agency. The main attributes include the agency id and Service Id, which are a compound PK. The table also has the name of the service along with Average, Minimum, and Maximum Processing Time of each service. The attribute "Agency Id" is a FK on Agencies, which contains the details of all government agencies. All these properties are listed in Table 1.

Table 1 The structure of services table

\begin{tabular}{ll}
\hline Table & Attribute (Property/Relation) \\
\hline Services & Agency Id (PK + FK on Agencies) \\
& Service Id (PK) \\
& Name \\
& Average Processing Time \\
& Minimum Processing Time \\
& Maximum Processing Time \\
\hline
\end{tabular}

\subsection{Inputs}

This is a lookup table that contains the details of all inputs required by the customers when requesting the services. The main attributes are the Input Id, which is the PK, the Name of the input, and its Description. All these properties are listed in Table 2.

Table 2 The structure of inputs table

\begin{tabular}{ll}
\hline Table & Attribute (Property/Relation) \\
\hline Inputs & Input Id (PK) \\
& Name \\
& Description \\
\hline
\end{tabular}

\subsection{0utcomes}

This is a lookup table that contains the details of all outcomes that any service/step can produce. The main attributes are the Outcome Id, which is a PK, the Name of the outcome, and its Description. All these properties are listed in Table 3.

27
Table 3 The structure of Outcomes table

\begin{tabular}{ll}
\hline Table & Attribute (Property/Relation) \\
\hline Outcomes & Outcome Id (Pk) \\
& Name \\
& Description \\
\hline
\end{tabular}

\subsection{Steps}

This table contains the details of all steps of each service. The main attributes include the Service Id and the Step Id, which are a compound PK. The Service Id is a FK on Services. The table also has the Step Name along with Average, Minimum, and Maximum Processing Time of each step. The step sequence attributes determine the sequence of the step in each service. All these properties are listed in Table 4.

Table 4 The structure of steps table

\begin{tabular}{ll}
\hline Table & Attribute (Property/Relation) \\
\hline Steps & Service Id (PK + FK on Services) \\
& Step Id (PK) \\
& Step Name \\
& Step Sequence \\
& Average processing time \\
& Minimum processing time \\
& Maximum processing time \\
\hline
\end{tabular}

4.5Steps inputs

This table contains the details of the inputs of each step in each service. The main attributes of this table are the Service Id, Step Id, and Input Id, which are a compound PK that prevents redundant inputs per steps. Service Id \& Step Id are a compound FK on Steps and Input Id is a FK on Inputs. All these properties are listed in Table 5.

Table 5 The structure of steps inputs table

\begin{tabular}{ll}
\hline Table & Attribute (Property/Relation) \\
\hline Steps Inputs & Service Id (PK + FK on Steps) \\
& Step Id (PK + FK on Steps) \\
& Input Id (PK + FK on Inputs) \\
\hline
\end{tabular}

\subsection{Steps outcomes}

This table contains the details of the outcomes of each step in each service. The main attribute of this table are the Service Id, Step Id, and Outcome Id, which are a compound PK that prevents redundant outputs per steps. Service Id \& Step Id are a compound FK on Steps and Outcome Id is a FK on Inputs. All these properties are listed in Table 6.

Table 6 The structure of steps outcomes table

\begin{tabular}{ll}
\hline Table & Attribute (Property/Relation) \\
\hline Steps Outcomes & Service Id (PK + FK on Steps) \\
& Step Id (PK + FK on Steps) \\
& Outcome Id (PK + FK on Inputs) \\
\hline
\end{tabular}




\begin{tabular}{ll}
\hline Table & Attribute (Property/Relation) \\
\hline & Justification \\
\hline
\end{tabular}

\subsection{Checklists}

This table contains the details of the items that each step should pass in order to progress to the next step. The main attributes of this table include the Service Id, Step Id, and the Item Id, which are a compound PK. Both the Service Id and the Step Id are a compound FK on Steps. The table also has the Item Name, and Description along with Average, Minimum, and Maximum Processing Time of each item. All these properties are listed in Table 7.

Table 7 The structure of checklists table

\begin{tabular}{ll}
\hline Table & Attribute (Property/Relation) \\
\hline Checklists & Service Id (PK + FK on Steps) \\
& Step Id (PK + FK on Steps) \\
& Item Id (PK) \\
& Item Name \\
& Item Description \\
& Item Sequence \\
& Average Processing Time \\
& Minimum Processing Time \\
& Maximum Processing Time \\
\hline
\end{tabular}

\subsection{Employees responsibilities}

This table contains the responsibilities of each employee in the government agency. Each employee can be assigned one or more steps from the same or different services. The main attributes of this table are the Employee Id, Service Id and Step Id, which are a compound PK. This guarantees the uniqueness of steps, assignment per employee and it allows assigning any step to more than one employee. The Employee Id is a FK on Employees (it is assumed that this table exists in the database, which contains the information of all agency employees). The Service Id and Step Id are a compound FK on Steps, which guarantee the integrity of the data in this table. All these properties are listed in Table 8.

Table 8 the structure of employees responsibilities table.

\begin{tabular}{|c|c|}
\hline Table & $\begin{array}{l}\text { Attribute } \\
\text { (Property/Relation) }\end{array}$ \\
\hline Employees & Employee Id (PK + FK on \\
\hline \multirow{2}{*}{ Responsibilities } & Employees) \\
\hline & $\begin{array}{l}\text { Service Id }(\mathrm{PK}+\mathrm{FK} \text { on } \\
\text { Steps }) \\
\text { Step Id (PK + FK on Steps })\end{array}$ \\
\hline
\end{tabular}

\subsection{Processed Services}

This table contains the details of all requested services. The main attribute of this table are the Customer Id, Service Id, Employee Id, and the 28
Request Timestamp. There is no PK in this table because any customer can request the same service more than one time. In order to guarantee the integrity of the data in this table, a number of FKs have to be created as follows. The Customer Id refers to Customers (a table that is assumed to exist, which contains the information of all customers). The Service Id refers to the services and the employee id refers to employees. All these properties are listed in Table 9.

Table 9 The structure of processed services table.

\begin{tabular}{ll}
\hline Table & Attribute (Property/Relation) \\
\hline Processed Services & Customer Id (FK on Customers) \\
& Service Id (FK on steps) \\
& Step Id (FK on steps) \\
& Employee Id (FK on Employees) \\
& Submission Timestamp \\
& Outcome Id (FK on outcomes) \\
& Completion Timestamp \\
\hline
\end{tabular}

\section{Data analysis techniques}

This section proposes a number of data analysis techniques that can be utilized to enhance the quality of customer service in government sectors. The proposed techniques assume the existence of a CSIS that have a database structure similar to the one described above. Each of the following subsections proposes a data analysis technique that efficiently utilizes the existing data in CSIS to give the decision maker a good reading on how to improve the quality of customer service or improve the existing services. The text below provides the equations and approaches required to develop such data analysis techniques while the select statements/algorithmic implementations are left due to their simplicity.

5.1Reviewing the structure of the services/steps It is important that the government agency (service provider) regularly reviews the structure of its services to improve their quality. The reviewing process suggests either changing the structure of the service or keeping it as is. Changing the structure includes adding one or more steps and/or removing one or more steps. One of the vital tools for reviewing service structure is to statistically summarize the historical data of each step in the service to find its success rate. The success rate can be calculated from equation (1):

$$
S S R=\frac{\sum S S}{\sum P S}
$$

, where $S S R=$ Step Successful Rate $\sum S S=$ Total number of Successful Steps 


\section{$\sum P S=$ Total number of Processed Steps}

From a statistical point of view, the successful rate of steps should increase as the service advances through the steps. If any step has a success rate less than its predecessor, this suggests moving that step before the first step that has a higher success rate [2-8]. If that is impossible due to rules and regulations, then the checklist that causes this decreasing in success rate might be moved earlier.

\subsection{Determining services/steps timescale}

Professional service providers provide their customers with the time required for each service/step to be processed. For instance, the Ministry of Commerce and Industry (MCI) announced that the processing of a commercial registration takes 180 seconds for individual institutions. Determining the processing time is easy only if the service is completely automated and does not require human intervention (NHIS) [3-5]. However, if the service is a HIS, then its processing time depends upon human and differs from one employee to another or even from time to time. In this case, it has to be treated statistically. For this reason, the database structure proposed above stores the submission and the completion timestamp of each processed step. This allows determining the average, minimum, and maximum processing time for each service/step. These values can be calculated from equations (2), (3), and (4), respectively.

$$
\begin{gathered}
A P T=\frac{\sum(C T-S T)}{\sum P S} \\
M i n P T=\min \left(C T_{1}-S T_{1}, C T_{2}\right. \\
\left.-S T_{2}, \ldots, C T_{n}-S T_{n}\right) \\
M a x P T=\max \left(C T_{1}-S T_{1}, C T_{2}\right. \\
\left.-S T_{2}, \ldots, C T_{n}-S T_{n}\right)
\end{gathered}
$$

, where $A P T=$ Average Processing Time

MinPT $=$ Minimum Processing Time

MaxPT $=$ Maximum Processing Time

$C T=$ Completion Timestamp

$S T=$ Submission Timestamp

\subsection{Administering human resource $(\mathrm{HR})$}

It is important that the government agencies make sure that services/steps do not halt or left without assignment [1-2]. In order to guarantee that, the service provider has to make sure that any requested service/step has the enough HR to process it. Mathematically, enough HR means the total working hours of all employees who are free \& authorized to process services/steps is more than or at least equal to the average processing hours of all active relevant services/steps. This can be calculated from equation (5).

$$
\begin{aligned}
& \sum(W H \times A E) \geq \sum(A P T \times A S) \quad \text { (5) } \\
& \text {, where } \\
& W H=\text { Daily working Hours } \\
& A E=\text { Number of Available Employees who } \\
& \text { are free and authorized to process the } \\
& \text { services/steps } \\
& A S=\text { Active Services/Steps }
\end{aligned}
$$

\subsection{Determining the efficiency of $H R$}

The main processor for all services/step is the HR. Therefore, it is a wise approach to assess HR based on the quality of processing services/steps. Two main metrics can be utilized for this purpose:

\subsubsection{Processing timescale}

Good employees should process services/steps in an amount of time that is very near to the average processing time and is less than the maximum processing time. Outstanding employees are expected to process services/steps in an amount of time that is very near to or less than the minimum process time.

5.4.2Processing quality

Processing quality means taking the right decision. Thus, it can be calculated from the total number of services/steps that are not returned to the employee as incomplete or wrongly processed.

5.5Reducing the number of refused services/steps Professional service providers save their employees and customer's time via decreasing the number of refused services/steps, especially those refused due to missing/wrong documents/information. Statistical analysis of historical data can be summarized to give the customers important information about the most likely reasons that cause refusing the services/steps. This will increase the awareness of the customers so that they take extra care about the inputs that might cause refusing the services/steps. Hence, decreasing the number of refused services/steps.

\subsection{Reducing the storage size of CSIS}

Some inputs are common between several services/steps. Accordingly, it will save the size of the system if these documents are stored in the customer profiles and then the customers refer to these documents when applying for services.

\section{Conclusion and future work}

It is the fact that all employees in all jobs around the world are working towards providing services to 
others. While each employee serves others, he/she receives services from others. Nowadays, processing services require an Information System (IS) that is called Customer Service Information System (CSIS). This system comprises a database in its backend and a number of forms in its frontend. Most existing CSISs automate the process of processing services but not utilize the available data to improve the quality of customer service. This paper addressed this gap by proposing a number of data analysis techniques that can be utilized to improve the quality of customer service and enhance the existing services. Moreover, this research paper introduced an efficient database structure that can be utilized to apply the proposed data analysis techniques.

The future work of this research might include statistical analysis of real data collected from Umm Al-Qura University (UQU) in order to give decision makers valuable readings and suggestions to improve the process of customer service in the university.

\section{Acknowledgment}

None.

\section{Conflicts of interest}

The author has no conflicts of interest to declare.

\section{References}

[1] Cook S. Customer care excellence: how to create an effective customer focus. Kogan Page Publishers; 2010.

[2] Ukens LL. 101 ways to improve customer service: training, tools, tips, and techniques. John Wiley \& Sons; 2007.

[3] Berndt A, Brink A. Customer relationship management and customer service. Juta and Company Ltd.; 2004.
[4] Wellington P. Effective customer care. Kogan Page Publishers; 2010.

[5] Hayes J, Dredge F. Managing customer service. Gower Publishing, Ltd.; 1998.

[6] Bestavros A, Fay-Wolfe V, editors. Real-time database and information systems: research advances: research advances. Springer Science \& Business Media; 2012.

[7] Sharp DE. Customer relationship management systems handbook. CRC Press; 2002.

[8] Kumar V, Reinartz W. Customer relationship management: a data based approach. John Wiley \& Sons; 2006.

[9] Sheth JN. Customer relationship management: emerging concepts, tools, and applications. Parvatiyar A, Shainesh G, editors. Tata McGraw-Hill Education; 2001.

[10] Buttle F. Customer relationship management:concepts and technologies. Routledge;2009.

\begin{abstract}
Abdullah Baz is an Assistant Professor of computer system design with the Computer Engineering Department, UQU. His research interests include VLSI design, EDA/CAD tools, coding and modulation schemes, image \& vision computing, computer system \& architecture, and digital signal processing. He has profound experience with various computer systems solutions including both software and hardware aspects. He has worked with several leading research communities to design, implement, and test various computing solutions for a number of national and international organizations. Currently, he is a reviewer committee member of the IEEE International Symposium on Circuits and Systems (ISCAS) \& IET Circuits, Devices $\&$ Systems and a member of the technical committee of IEEE VLSI systems and applications. $\mathrm{He}$ is also an editorial board member of the American Journal of Circuits, Systems and Signal Processing and the reviewer of Control Theory and Informatics.

Email: aobaz01@uqu.edu.sa
\end{abstract}

